SELECCIONES MATEMÁTICAS
Universidad Nacional de Trujillo
ISSN: $2411-1783$ (Online)
2021; Vol. 8(2): 379-385.

\title{
A modified Leslie-Gower-type predation model considering collaboration between predators
}

\section{Un modelo de depredación de tipo Leslie-Gower modificado que considera la colaboración entre depredadores}

\author{
Eduardo González-Olivares ${ }^{\circledR}$ and Alejandro Rojas-Palma (i)
}

Received, Jul. 30, 2021

Accepted, Dec. 15, 2021

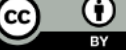

How to cite this article:

González-Olivares E, Rojas-Palma A. A modified Leslie-Gower-type predation model considering collaboration between predators. Selecciones Matemáticas. 2021;8(2):379-385. http://dx.doi .org/10.17268/sel .mat.2021. 02.14

\begin{abstract}
The interactions between predators and their prey are one of the most important aspects in the dynamics of food chains or trophic webs.

Usually, this relationship in the real world is influenced by various behaviors, both from prey and predators. Collaboration or cooperation between predators is one of those behaviors, which has received less attention than other behaviors of predators, such as competition between them.

In this work, we will model the cooperation between predators to capture (or consume) their favorite prey using a recent proposition that modifies the linear functional response of the Leslie-Gower model.

We show that this modified model has richer dynamics than the original, obtaining varied results. Among the main ones, populations can oscillate around a point where population sizes are fixed.

Keywords . Predator-prey model, functional response, stability, bifurcations, limit cycles.
\end{abstract}

\section{Resumen}

Las interacciones entre los depredadores y sus presas son uno de las aspectos más importantes en las dinámicas de las cadenas alimenticias o redes tróficas.

Usualmente esta relación en el mundo real es influenciada por diversos comportamientos, tanto de las presas como de los depredadores. La colaboración o cooperación entre los depredadores es una de esas conductas, la cual ha recibido menor atención que otros comportamientos de los depredadores, como por ejemplo la competición entre ellos.

En este trabajo, modelaremos la cooperación entre los depredadores para capturar (o consumir) a sus presas favoritas usando una reciente proposición que modifica la respuesta funcional lineal del modelo de Leslie-Gower.

Mostramos que este modelo modificado tiene dinámicas más ricas que el original, obteniendo variados resultados. Entre los principales se tiene que las poblaciones pueden oscilar alrededor de un punto donde los tamaños poblacionales son fijos.

Palabras clave. Modelo depredador-presa, respuesta funcional, estabilidad, bifurcaciones, ciclo límite.

1. Introducción. Las interacciones sociales entre individuos son un aspecto importante en la historia de vida de muchas especies. Los depredadores suelen competir [11] o colaborar al realizar la captura de sus presas [24]. Estos fenómenos pueden tener fuertes consecuencias en la relación entre ellos y modificar las propiedades dinámicas de un sistema que lo describe [11].

\footnotetext{
*Pontificia Universidad Católica de Valparaíso, Chile. (e jgonzal@ucv.cl).

†Departamento de Matemática, Física y Estadística, Facultad de Ciencias Básicas, Universidad Católica del Maule, Talca, Chile. (amrojas@ucm.cl)
} 
La caza en grupo (hunting collaboration [22]) es una forma de facilitar la búsqueda del alimento y puede inducir el fenómeno denominado efecto Allee en la población de presas [7]; pero también puede aumentar el riesgo de enfermedades o parásitos en la misma especie depredadora, la competencia por la comida y la atracción de otros depredadores (intermedios) o bien, servir como alimento para otros depredadores (depredadores top) [15].

Particularmente, la cooperación o colaboración entre depredadores es bastante frecuente en la naturaleza, representando un mecanismo desarrollado a través de la evolución para mejorar las habilidades de caza y las posibilidades de supervivencia. Por ejemplo, los lobos que siguen a los bisontes [18], las hienas que persiguen a los búfalos, los perros salvajes africanos (lincaones) en busca de cebras [15], estrellas de mar consumiendo una comunidad de arrecifes de coral [1, 2], etc.

En este trabajo, para describir la caza colaborativa, se asumirá que la respuesta funcional es dependiente tanto de la densidad de presas como de depredadores. Supondremos que los depredadores cooperadores se benefician de su comportamiento, por lo que el éxito de los ataques a las presas aumenta con la densidad de depredadores. Siguiendo lo expuesto en [22], esta suposición se representa en el modelo reemplazando la tasa usual de ataque constante $q$, propuesta en el modelo de Leslie [16] y de Volterra [23], por un término dependiente del tamaño poblacional de los depredadores, dado por

$$
h(x, y)=(q+a y) x=q\left(1+\frac{a}{q} y\right) x,
$$

donde $x=x(t)$ e $y=y(t)$ indican los tamaños poblacionales de presas y depredadores, respectivamente, para $t \geq 0$; el parámetro $a>0$ describe una relación proporcional entre la cooperación de los depredadores en la caza de sus presas y el tamaño poblacional de los depredadores [22, 24]. El cuociente $\frac{a}{q} y$ es llamado el término de cooperación.

Es necesario señalar que existen otras formas matemáticas para expresar el comportamiento de colaboración entre los depredadores para capturar sus presas [14] y que la comparación de los distintos modelos propuestos es necesaria.

Si $a=0$, tenemos un modelo depredador-presa sin cooperación en la caza o captura de sus presas.

Observamos que si $a<0$, esto representaría la interferencia o competencia entre los depredadores al realizar la captura de sus presas; en este caso, se debe cumplir que el término $q+a y$ debe ser positivo también para representar realmente un modelo de depredación [23].

Sin embargo, otra interpretación es posible para la función $h(x, y)$ cuando $a<0$, asumiendo que una fracción $x_{r}$ de la población de presas hace uso de un refugio físico [19]. Entonces, el tamaño de la población disponible para ser consumida por los depredadores es $x-x_{r}$ [19].

Suponiendo que la población a cubierto es proporcional al encuentro entre ambas especies, se tiene $x_{r}=\sigma x y, \operatorname{con} \sigma>0$ [10]. Entonces, la respuesta funcional lineal es descrita ahora por

$$
h(x, y)=q\left(x-x_{r}\right) y=q(x-\sigma x y) y=q(1-\sigma y) x y, \text { con } \sigma=-\frac{a}{q} .
$$

El estudio del efecto del uso de refugio proporcional a los encuentros por parte de las presas, es motivo de análisis en otro trabajo [20].

Este trabajo está organizado de la siguiente manera: En la Sección 2 describiremos el modelo y en la Sección 3 expondremos las propiedades fundamentales del sistema planar que describe el modelo. En la Sección 4, discutimos las propiedades del modelo y las interpretamos desde un punto de vista ecológico.

2. Proposición del modelo. El modelo a estudiar es una modificación del modelo de Leslie-Gower $[16,17]$, siendo descrito por el siguiente sistema bidimensional de ecuaciones diferenciales autónomas del tipo Kolmogorov [8, 9]:

$$
X_{\mu}(x, y):\left\{\begin{aligned}
\frac{d x}{d t} & =\left(r\left(1-\frac{x}{K}\right)-q\left(1+\frac{a}{q} y\right) y\right) x \\
\frac{d y}{d t} & =s\left(1-\frac{y}{n x+c}\right) y
\end{aligned}\right.
$$

donde $x=x(t)$ e $y=y(t)$ son los tamaños poblacionales de presas y depredadores, respectivamente, para $t \geq 0$, con $\mu=(r, K, q, a, s, n, c) \in \mathbb{R}_{+}^{7}$, teniendo los significados ecológicos:

$r$ y $s$ indican la tasa de crecimiento intrínseca de la población de presas y depredadores, respectivamente,

$K$ es la capacidad de carga ambiental de la presa,

$q$ es la tasa de consumo de depredadores,

$n$ representa la calidad energética como alimento que la presa proporciona a los depredadores,

$c$ indica el tamaño máximo disponible del alimento alternativo, 
$\alpha$ es la tasa de cooperación depredadora en la caza constante.

El sistema (2.1) está definido en todo el primer cuadrante, es decir, definido en el conjunto $\Omega=\left\{(x, y) \in \mathbb{R}^{2}: x \geq 0, y \geq 0\right\}$.

Los puntos de equilibrio del sistema (2.1) o singularidades del campo vectorial $X_{\mu}(x, y)$ son: $(0,0)$, $(K, 0),(0, c), \mathrm{y}$ los puntos $\left(x_{e}, y_{e}\right)$ que están en la intersección de las isoclinas

$$
y=n x+c \text { and } r\left(1-\frac{x}{K}\right)-q\left(1+\frac{a}{q} y\right) y=0,
$$

o sea, la absisa $x_{e}$ satisface la ecuación cuadrática,

$$
P(x)=\left(K a n^{2}\right) x^{2}+(r+K n q+2 K a c n) x+K\left(-r+a c^{2}+c q\right)=0,
$$

la cual tiene una raíz real positiva, si y sólo si, $r>c(q+a c)$.

A su vez, la ordenada $y_{e}$ satisface la ecuación,

$$
P(y)=K a n y^{2}+(r+K n q) y-r(c+K n)=0,
$$

que tiene una única raíz real positiva dada por:

$$
y_{e}=\frac{1}{2 K a n}(-(r+K n q)+\sqrt{\Delta})
$$

con

$$
\begin{aligned}
\Delta & =(r+K n q)^{2}+4 K a n r(c+K n) \\
& =r^{2}+2 K n(q+2 a c+2 K a n) r+K^{2} n^{2} q^{2} .
\end{aligned}
$$

Esto nos asegura la existencia de un único punto de equilibrio positivo $\left(x_{e}, n x_{e}+c\right)$, si y sólo si, $r>$ $c(q+a c)$.

Notamos que las coordenadas del punto de equilibrio positivo no dependen del parámetro $s$.

Para determinar la naturaleza de los puntos de equilibrio hiperbólicos o genéricos se usa la matriz Jacobiana dada por

$$
D X_{\mu}(x, y)=\left(\begin{array}{cc}
-\frac{1}{K}\left(K a y^{2}+K q y-K r+2 r x\right) & -x(q+2 a y) \\
n s \frac{y^{2}}{(n x+c)^{2}} & \frac{s}{n x+c}(c-2 y+n x)
\end{array}\right) .
$$

Mostraremos que el modelo considerando colaboración en la captura de las presas, descrito por el sistema de ecuaciones diferenciales no lineales (1) tiene una dinámica más variada que la del modelo de Leslie original [16] y del modelo derivado en el cual los depredadores disponen de un alimento alternativo [13].

3. Resultados principales. Para el sistema (2.1) o campo vectorial $X_{\mu}(x, y)$ tenemos las siguientes propiedades

Lema 3.1. Existencia de una región invariante

El conjunto $\Gamma=\{(x, y) \in \Omega: 0 \leq x \leq K, y \geq 0\}$ es una región positivamente invariante.

Demostración: El sistema (1) es de tipo Kolmogorov y los ejes son conjuntos invariantes.

Asumiendo $x=K$, se tiene que:

La componente horizontal del campo vectorial tiene dirección negativa para $x \in[0, K]$.

$$
\frac{d x}{d t}=-q\left(1+\frac{a}{q} y\right) y K<0
$$

Cualquiera sea el signo de $\frac{d y}{d t}=s\left(1-\frac{y}{n x+c}\right) y$, se obtiene que toda solución que ingresa a $\Gamma$ no puede salir de allí.

Observación 3.1. Observamos que el conjunto

$\Lambda=\{(x, y) \in \Omega: 0 \leq x \leq K, 0 \leq y \leq n x+c\}$, es una región positivamente invariante, pues $\frac{d y}{d t}<0$, para todo $y>n x+c$ y las soluciones entran al conjunto $\Lambda$.

Además, $\Lambda$ es una región compacta donde aplica el Teorema de Bendixson-Poincaré.

Lema 3.2. Acotamiento de las soluciones

Las soluciones son uniformemente acotadas

Demostración: Usando el Teorema de Comparación para las desigualdades diferenciales [5]. 
De la primera ecuación tenemos

$\frac{d x}{d t} \leq r\left(1-\frac{x}{K}\right) x$, para todo $y \geq 0$, cuando $0<x<K$.

También sabemos que

$x \rightarrow K$, cuando $t \rightarrow \infty$ y $x<K$.

$x \rightarrow K$, cuando $t \rightarrow \infty$ y $x>K$.

Sea $L=\max \{x(0), K\}$; entonces $x(t) \leq L$, para todo $t \geq 0$.

Además, $0<y<n x+c$.

Considerando la nueva variable $w(x, y)=x+\frac{1}{s} y$, tenemos que, $w(x, y)>0$, para todo $t \geq 0$.

Por lo tanto,

$$
\frac{d w}{d t}=\frac{d x}{d t}+\frac{1}{s} \frac{d y}{d t}=\left(r\left(1-\frac{x}{K}\right)-q\left(1+\frac{a}{q} y\right) y\right) x+\left(1-\frac{y}{n x+c}\right) y .
$$

Ahora considerando la suma de $\frac{d w}{d t}$ y $\sigma w, \operatorname{con} \sigma>0$, obtenemos:

Luego

$$
\frac{d w}{d t}+\sigma w=r\left(1-\frac{x}{K}\right) x-q x\left(1+\frac{a}{q} y\right) y+\left(1-\frac{y}{n x+c}\right) y+\sigma x+\frac{\sigma}{s} y .
$$

$$
\begin{aligned}
& \frac{d w}{d t}+\sigma w=r x-\frac{r x^{2}}{K}-q x y-a x y^{2}+y-\frac{y^{2}}{n x+c}+\sigma x+\frac{\sigma}{s} y, \\
& \frac{d w}{d t}+\sigma w \leq(r+\sigma) x+\left(1+\frac{\sigma}{s}\right) y-a x y^{2}, \\
& \frac{d w}{d t}+\sigma w \leq(r+\sigma) x-(a x)\left(y^{2}-\frac{\left(1+\frac{\sigma}{s}\right)}{a x} y+\frac{1}{4}\left(\frac{\left(1+\frac{\sigma}{s}\right)}{a x}\right)^{2}\right)+(a x) \frac{1}{4}\left(\frac{\left(1+\frac{\sigma}{s}\right)}{a x}\right)^{2}, \\
& \frac{d w}{d t}+\sigma w \leq(r+\sigma) x-(a x)\left(y-\frac{\left(1+\frac{\sigma}{s}\right)}{2 a x}\right)^{2}+(a x) \frac{1}{4}\left(\frac{\left(1+\frac{\sigma}{s}\right)}{a x}\right)^{2}, \\
& \frac{d w}{d t}+\sigma w \leq(r+\sigma) x+(a x) \frac{1}{4}\left(\frac{\left(1+\frac{\sigma}{s}\right)}{a x}\right)^{2}, \\
& \frac{d w}{d t}+\sigma w \leq(r+\sigma) L+(a L) \frac{1}{4}\left(\frac{\left(1+\frac{\sigma}{s}\right)}{a L}\right)^{2} .
\end{aligned}
$$

Sea $N=(r+\sigma) L+(a L) \frac{1}{4}\left(\frac{\left(1+\frac{\sigma}{s}\right)}{a L}\right)^{2}$;

luego

$$
0<\frac{d w}{d t}+\sigma w \leq N
$$

que es una desigualdad diferencial de primer orden.

Aplicando el teorema de comparación para desigualdades diferenciables (página 30 en [5]), obtenemos que

$$
0<w(t) e^{\tau} \leq N e^{t}+N_{2} .
$$

Cuando $t=0$, obtenemos que $w(0) \leq N+N_{2}$; o sea, $N_{2} \geq w(0)-N$.

Entonces hay $p \in \mathbb{N}$ tal que $N_{2} \leq p(w(0)-N)$.

Por lo tanto, $w(t) e^{t} \leq N e^{t}+p(w(0)-N)$,

i.e., $w(t) \leq N+p(w(0)-N) e^{-t}$.

Luego, cuando $t \rightarrow \infty$ entonces $w(t) \leq N$.

Por tanto, las soluciones están acotadas.

Lema 3.3. Naturaleza de los puntos de equilibrio sobre el eje $x$.

1. El punto $(0,0)$ es un repulsor hiperbólico.

2. El punto $(K, 0)$ es una silla hiperbólica.

Demostración: Es inmediata pues evaluando la matriz Jacobiana, se tiene:

1.

$$
D X_{\mu}(0,0)=\left(\begin{array}{cc}
r & 0 \\
0 & s
\end{array}\right),
$$

con $\operatorname{det} D Y_{\nu}(0,0)=r s>0 \mathrm{y} \operatorname{tr} D Y_{\nu}(0,0)=r+s>0$.

2 .

$$
D X_{\mu}(K, 0)=\left(\begin{array}{cc}
-r & -K q \\
0 & s
\end{array}\right),
$$

con $\operatorname{det} D X_{\mu}(K, 0)=-r s<0$.

Lema 3.4. Naturaleza del punto de equilibrio sobre el eje y.

El punto $(0, c)$ es:

i) Una silla hiperbólica, si y sólo si, $r>c(q+a c)$,

ii) Un atractor hiperbólico, si y sólo si, $r<c(q+a c)$,

iii) Un silla-nodo atractor (no-hiperbólico), si y sólo si, $r=c(q+a c)$. 
Demostración: Evaluando la matriz Jacobiana tenemos

$$
D X_{\mu}(0, c)=\left(\begin{array}{cc}
r-a c^{2}-c q & 0 \\
n s & -s
\end{array}\right)
$$

con $\operatorname{det} D X_{\mu}(0, c)=-s\left(r-a c^{2}-c q\right)$.

i) Si $r-a c^{2}-c q>0$, entonces $\operatorname{det} D X_{\mu}(0, c)<0$ y $(0, c)$ es punto silla hiperbólica.

ii) Si $r-a c^{2}-c q<0$, entonces $\operatorname{det} D X_{\mu}(0, c)>0$ y $\operatorname{tr} D X_{\mu}(0, c)=r-a c^{2}-c q-s<0$, luego $(0, c)$ es un punto de equilibrio atractor hiperbólico.

iii) Si $r-a c^{2}-c q=0$, entonces $\operatorname{det} D X_{\mu}(0, c)=0 \mathrm{y} \operatorname{tr} D X_{\mu}(0, c)=-s<0$.

Teorema 3.1. Naturaleza del punto de equilibrio positivo.

El punto de equilibrio positivo $\left(x_{e}, n x_{e}+c\right)$ es:

i) Un repulsor, si y sólo si, $s<\frac{x_{e}\left(-r+2 K_{a n}^{2} x_{e}\right)}{K}$, y existe un ciclo límite,

ii) Un atractor, si y sólo si, $s>\frac{x_{e}\left(-r+2 K_{a n}^{2} x_{e}\right)}{K}$,

iii) Un foco débil, si y sólo si, $s=\frac{x_{e}\left(-r+2 K a n^{2} x_{e}\right)}{K}$.

Demostración: La matriz Jacobiana evaluada en $\left(x_{e}, n x_{e}+c\right)$ es

$$
D X_{\mu}\left(x_{e}, n x_{e}+c\right)=\left(\begin{array}{cc}
D X_{\mu}\left(x_{e}, n x_{e}+c\right)_{11} & -x_{e}\left(q+2 a c+2 a n x_{e}\right) \\
n s & -s
\end{array}\right),
$$

$\operatorname{con} D X_{\mu}\left(x_{e}, n x_{e}+c\right)_{11}=\frac{-K a n^{2} x^{2}+(-2 r-K n q-2 K a c n) x+K\left(r-q c-a c^{2}\right)}{K}$.

Se tiene que

$\operatorname{det} D X_{\mu}\left(x_{e}, n x_{e}+c\right)=\frac{s}{K}\left(3 K_{a n}^{2} x_{e}^{2}+2(r+K n q+2 K a c n) x_{e}+K\left(-r+a c^{2}+c q\right)\right)$.

Como $x_{e}$ satisface la ecuación (2), reemplazando se obtiene

$$
\operatorname{det} D X_{\mu}\left(x_{e}, n x_{e}+c\right)=s x_{e} \frac{2 K a n^{2} x_{e}+(r+K n q+2 K a c n)}{K}>0 .
$$

Por lo tanto, la naturaleza del punto depende de la traza.

$$
\operatorname{tr} D X_{\mu}\left(x_{e}, n x_{e}+c\right)=\frac{-K a n^{2} x_{e}^{2}+(-2 r-K n q-2 K a c n) x_{e}+K\left(r-q c-a c^{2}\right)}{K}-s .
$$

Recordando que $x_{e}$ satisface la ecuación (2), reemplazando se obtiene

Luego,

$$
\operatorname{tr} D X_{\mu}\left(x_{e}, n x_{e}+c\right)=\frac{x_{e}\left(-r+2 \operatorname{Kan}^{2} x_{e}\right)}{K}-s .
$$

$$
\operatorname{tr} D X_{\mu}\left(x_{e}, n x_{e}+c\right)>0 \text {, si y sólo si, } s<\frac{x_{e}\left(-r+2 \operatorname{Kan}^{2} x_{e}\right)}{K},
$$

ii) $\operatorname{tr} D X_{\mu}\left(x_{e}, n x_{e}+c\right)>0$, si y sólo si, $s>\frac{x_{e}\left(-r+2 \operatorname{Kan}^{2} x_{e}\right)}{K}$,

iii) $\operatorname{tr} D X_{\mu}\left(x_{e}, n x_{e}+c\right)=0$, si y sólo si, $s=\frac{x_{e}\left(-r+2 K a n^{2} x_{e}\right)}{K}$.

Entonces, se cumple el enunciado.

\section{Corolario 3.1 (Existencia de una bifurcación de Hopf).}

Existe al menos un ciclo límite alrededor del punto $\left(x_{e}, n x_{e}+c\right)$.

Demostración: Cuando el punto $\left(x_{e}, n x_{e}+c\right)$ es repulsor, de acuerdo con el teorema de PoincaréBendixson, debe existir un ciclo límite.

La existencia de la bifurcación de Hopf es inmediata, pues la condición de transversalidad [6] implica que

$$
\frac{\partial}{\partial s}\left(\operatorname{tr} D Y_{\mu}\left(x_{e}, n x_{e}+c\right)\right)=-1<0 .
$$

4. Conclusiones. En este trabajo, hemos estudiado una modificación del modelo de Leslie-Gower $[16,17]$, considerando la colaboración entre los depredadores, ampliando los resultados del modelo de Leslie-Gower [16, 17].

Después de nuestro análisis, podemos resaltar los siguientes aspectos que lo diferencian del artículo original de Leslie-Gower:

1. Existe un punto de equilibrio sobre el eje vertical, que puede ser un punto silla o un punto atractor hiperbólico o no-hiperbólico, según determinadas condiciones del espacio de parámetros.

2. Hay un subconjunto de valores de parámetros para los cuales la línea recta $y=n x+c$ determina una región cuadrangular invariante con los ejes de coordenadas.

3. Una bifurcación de Hopf es generada en el único punto de equilibrio positivo dando origen a un ciclo límite estable.

La inclusión de la colaboración o cooperación entre los depredadores para optimizar la captura de sus presas, descrito por el parámetro $a>0$, influye fuertemente en las propiedades matemáticas del sistema EDO que describe el modelo. Al incluir este comportamento social de los depredadores, el sistema tiene 
dinámicas más ricas e interesantes que el modelo de Leslie-Gower [23] en el cual nos hemos basado para incluirlo.

En el artículo de Berryman et al. [4] se expresa que: "Además de una base mecanicista lógica, un modelo depredador-presa creíble también debe poseer propiedades biológicas y ecológicas razonables". En ese trabajo, se establecen los atributos mínimos para que un modelo depredador-presa sea creíble. Luego, estos atributos determinan varias condiciones para la ecuación de las presas y diferentes condiciones para los parámetros.

Nosotros consideramos que el modelo estudiado satisface esos requerimientos y que las interpretaciones ecológicas de los resultados analíticos obtenidos tienen coherencia y son congruentes con los supuestos declarados.

La cooperación en la caza por parte de los depredadoress puede tener efectos no solo positivos, sino también negativos para ellos. Si la colaboración es demasiado intensa, implica que la densidad de equilibrio de depredadores disminuye con un mayor aumento de la tasa de cooperación.

Mostramos que demasiada cooperación (cuando $a \rightarrow \infty$ ) puede ser contraproducente para los depredadores, ya que pueden sobreexplotar a sus presas; se tiene que el punto $(0, c)$ puede ser atractor para cierta condición de los parámetros.

Consideramos que las oscilaciones de las poblaciones descritas por el ciclo límite obtenido para cierto subconjunto de parámetros, pueden ser inducidas únicamente por las posibilidades de mejoría en la alimentación, debido a la cooperación, porque nuestro modelo se basa en la respuesta funcional lineal que no produce oscilaciones si no se considera la cooperación para la caza.

La facilidad para conseguir alimento puede ayudar en la supervivencia de la población de los depredadores. Los depredadores se extinguirían más facilmente sin cooperación en la caza; en el modelo de Leslie-Gower es posible que el punto $(K, 0)$ sea un atractor.

Sin embargo, es posible que los depredadores no se beneficien de la caza colaborativa de alimento indefinidamente, porque en algún momento el éxito de los ataques no podría aumentar con el aumento de la densidad de depredadores. En cambio, la interferencia o competencia entre los depredadores podría ayudar a mejorar las posibilidades de la alimentación en densidades más grandes.

Para un futuro trabajo queda pendiente determinar la debilidad del foco débil o probar que el ciclo límite en el sistema es único.

En base al modelo estudiado podemos derivar nuevos modelos de depredación, en los cuales se asuman los siguientes fenómenos ecológicos:

- La población de presas es afectada por un efecto Allee [7, 22].

- Una fracción de la población de presas hace uso de refugio [19].

- La acción del depredador es descrita por una respuesta funcional no-lineal [3, 21].

- La colaboración entre los depredadores es saturada [12].

ORCID and License

Eduardo González-Olivares https://orcid.org/0000-0003-3907-0076

Alejandro Rojas-Palma https://orcid.org/0000-0002-5837-1571

This work is licensed under the Creative Commons - Attribution 4.0 International (CC BY 4.0)

\section{Referencias}

[1] Antonelli PL, Kazarinoff ND. Starfish predation of a growing coral reef community. J. Theor. Biol.. 1984;107:667-684.

[2] Antonelli PL, Kazarinoff ND. Modelling density-dependent aggregation and reproduction in certain terrestrial and marine ecosystems: A comparative study, Ecological Modelling 1988;41:219-227.

[3] Bazykin AD. Nonlinear Dynamics of interacting populations, World Scientific Publishing Co. Pte. Ltd., Singapore 1998.

[4] Berryman AA, Gutierrez AP, Arditi R. Credible, parsimonious and useful predator-prey models - A reply to Abrams, Gleeson and Sarnelle, Ecology. 1995 76:1980-1985.

[5] Birkhoff G, Rota GS. Ordinary Differential Equations (4th ed.). John Wiley \& Sons, New York 1989.

[6] Chicone C. Ordinary differential equations with applications (2nd edition), Texts in Applied Mathematics 34, Springer 2006.

[7] Courchamp F, Berec L, Gascoigne J. Allee effects in Ecology and Conservation, Oxford University Press 2007.

[8] Freedman HI. Deterministic Mathematical Model in Population Ecology, Marcel Dekker 1980.

[9] Goh B-S. Management and Analysis of Biological Populations, Elsevier Scientific Publishing Company, 1980.

[10] González-Olivares E, González-Yañez B, Becerra-Klix R. Prey refuge use as a function of predator-prey encounters, Private communication, submitted to International Journal of Biomathematics (2012).

[11] González-Olivares E, Cabrera-Villegas J, Córdova-Lepe F, Rojas Palma A. Competition among predators and Allee effect on prey: their influence on a Gause-type predation model, Mathematical Problems in Engineering, vol. 2019, Article ID 3967408, 19 pages, 2019.

[12] González-Olivares E, Valenzuela-Figueroa S. and Rojas-Palma A. A simple Gause type predator-prey model considering social predation, Mathematical Methods in the Applied Sciences 42 (2019) 5668-5686. 
[13] González-Olivares E, Rojas Palma A. Influencia del efecto Allee fuerte en las presas y de la competición entre los depredadores en modelos de depredación del tipo Leslie-Gower, Selecciones Matemáticas 7(2) (2020) 302-313.

[14] González-Olivares E, Rojas-Palma A. Periodicidad en modelos de depredación del tipo Gause considerando colaboración entre los depredadores, Seleccions Matemáticas; 2021; 8(2):267-273.

[15] Hilker FM, Paliaga M, Venturino E. Diseased social predators, Bulletin of Mathematical Biology 2017;79:2175-2196.

[16] Leslie PH. Some further notes on the use of matrices in population mathematics, Biometrica 35 (1948) 213-245.

[17] Leslie PH, Gower JC. The properties of a stochastic model for the predator-prey type of interaction between two species, Biometrika 47 (1960) 219-234.

[18] MacNulty DR, Tallian A, Stahler DR, Smith DW. Influence of group size on the success of wolves hunting bison, Plos One 2014;9(11):e112884 1-8.

[19] Maynard Smith J. Models in Ecology, University Press 1974.

[20] Molla H, Rahman MS and Sarwardi S. Dynamical study of a prey-predator model incorporating nonlinear prey refuge and additive Allee effect acting on prey species. Model. Earth Syst. Environ. 7, 749-765 (2021).

[21] Taylor RJ. Predation, Chapman and Hall, 1984.

[22] Teixeira Alves M, Hilker FM. Hunting cooperation and Allee effects in predators, Journal of Theoretical Biology 419 (2017) 13-22.

[23] Turchin P. Complex Population Dynamics: A Theoretical/Empirical Synthesis, Princeton University Press, Princeton, New Jersey, 2003.

[24] Ye P, Wu D. Impacts of strong Allee effect and hunting cooperation for a Leslie-Gower predator-prey system, Chinese Journal of Physics 68 (2020) 49-64. 